\title{
Concevoir un parcours d'auto-apprentissage guidé de la prononciation du FLE sur Moodle
}

\author{
Emmanuelle Rassart \\ Université catholique de Louvain \\ Belgique
}

La compétence de prononciation est déterminante pour une communication orale fluide en langue étrangère (Dufeu 67), plus encore quand les apprenants sont en situation d'immersion linguistique, tels les étudiants et chercheurs internationaux quotidiennement confrontés aux interactions avec des natifs durant leur séjour dans une université francophone.

Dans La prononciation en classe, l'ouvrage co-publié aux PUG en 2014 avec Geneviève Briet et Valérie Collige, nous proposons une façon « décomplexée » d'enseigner la prononciation en classe, fondée sur une dynamique «corps-tête-c(h)œur». Notre approche privilégie l'appréhension et l'acquisition de la prononciation en groupe (chœur) dans une atmosphère détendue et dynamique (cœur), grâce notamment au jeu, aux gestes (corps) et à des explications théoriques simples (tête). Néanmoins, pour remédier aux difficultés très diverses dans l'acquisition de la prononciation, le présentiel atteint ses limites :

Il semble essentiel de se méfier des tenants de la solution phonétique : à chaque apprenant son évolution et sa solution adaptée. C'est plutôt à cette permanente attention sur les moyens variés de l'entrainement et au plaisir à produire la nouvelle langue que l'enseignant doit inviter l'apprenant, plutôt que d'imposer une méthode phonétique, qu'il n'aura souvent pas le loisir d'exploiter pleinement en classe. (Lauret 170)

Afin de permettre à chaque étudiant de poursuivre à son rythme les prises de conscience et le travail initiés en classe, une petite équipe de l'Université de Louvain (UCL) développe depuis septembre 2016 un parcours d'auto-apprentissage guidé de la prononciation du FLE sur Moodle, la plateforme d'apprentissage en ligne utilisée depuis plus de 10 ans à l'UCL.

Après avoir dressé l'état de la question, puis mis en évidence les besoins des étudiants, cet article développera les objectifs et les choix didactiques qui guident la conception du parcours en ligne. Quelques exemples illustreront le tout.

\section{Apprendre la prononciation en ligne : état de la question}

Travailler la prononciation grâce à des activités d'apprentissage en ligne permet de s'entraîner sans l'anxiété éventuellement générée par le regard des autres, y compris celui de l'enseignant. Pour Chapelle et Jamieson (159), exercer la prononciation face à un ordinateur - ou, en 2017, sur un téléphone portable ou une tablette - permet de développer des automatismes qui améliorent la fluidité. Les auteures soulignent également l'intérêt des applications d'entraînement basées sur la technologie de reconnaissance vocale $(\mathrm{RV})$, car celles-ci donnent un feedback immédiat à l'apprenant.

Dix ans après la formulation de cette recommandation, les technologies de RV ont fait d'énormes progrès en ce qui concerne la prise en charge des variétés géolinguistiques et prosodiques. Toutefois, leur utilité pédagogique pour l'apprentissage de la prononciation du français en autonomie reste encore à prouver. A ce jour, nous n'avons pas connaissance d'une application de RV qui prendrait en compte de façon satisfaisante la dimension suprasegmentale du français (intonation, place de l'accent, enchaînements, etc.). Dans leurs recherches sur l'impact des applications de RV sur téléphone mobile pour l'acquisition des phonèmes du français, Natallia Liakina et Denis Liakin pointent les mêmes limites : «Will other phonological or phonetic items such as features (e.g., spread glottis, voice-onset time), syllable structure (e.g., codas), rhythm and intonation benefit from a similar (mobile) ASR treatment ? » (Cardoso et al. 17 ; voir aussi Liakin et Liakina). Par ailleurs, à notre connaissance, la persistance dans la durée des progrès obtenus lors des séances d'entraînement phonétique avec technologie de RV n'a pas encore été mesurée. 
Au vu de ces limites technologiques, notre dispositif n'est pas conçu pour être utilisé en autonomie totale, ni pour donner un feedback automatisé sur la production des apprenants. II s'agit d'un dispositif complémentaire au travail de classe.

A notre connaissance, aucun projet similaire n'a encore vu le jour. Une première tentative d'apprentissage en ligne des principes de base de la prosodie et de la phonologie françaises avait été développée par Geneviève Briet pour le programme «Première classe »sur TV5MONDE¹. Les séquences consacrées à la prononciation proposaient des activités basées sur un document déclencheur. Nous reprenons certains canevas d'exercices dans notre dispositif Moodle. Pour terminer, il faut mentionner les expérimentations de quelques enseignants qui développent, dans leurs institutions respectives, des dispositifs d'entrainement de la prononciation du français accessibles en interne à leurs étudiants².

\section{Analyse des besoins}

\subsection{Public}

Le Département de français de l'Institut des langues vivantes de l'UCL offre une quinzaine de cours différents à environ 700 étudiants internationaux par an, depuis le niveau A1 jusqu'au C1. Le public se compose essentiellement d'étudiants d'échange et de chercheurs (doctorants et post-doctorants).

Sur le plan des nationalités, les Italiens et les Espagnols sont les plus nombreux, suivis des Chinois et des Allemands. L'ILV accueille des étudiants de presque tous les pays d'Europe grâce au programme de mobilité Erasmus, ainsi que des étudiants issus des pays voisins de l'UE (essentiellement Turquie et Russie). Enfin notre public compte de nombreux Asiatiques (surtout des Japonais, Vietnamiens et Indiens) et des SudAméricains.

Plus de $20 \%$ de nos étudiants ont pour langue maternelle l'espagnol, près de $20 \%$ l'italien, et $10 \%$ parlent d'abord le mandarin. L'anglais est pour beaucoup la lingua franca, même si moins de $5 \%$ de nos étudiants en sont des native speakers.

Les cours durent 13 semaines, conformément au calendrier académique de la Communauté française de Belgique. Ils comptent 2 ou 4 heures de présentiel par semaine. La majorité des étudiants ne séjournent qu'un semestre à Louvain-la-Neuve, ce qui restreint la possibilité de les accompagner dans leur apprentissage du français sur une période plus longue.

\subsection{Analyse de productions orales}

Afin de définir les objectifs du dispositif d'apprentissage sur Moodle, Geneviève Briet et moi-même sommes parties des évaluations diagnostiques de la prononciation de nos étudiants réalisées ces dernières années en oral spontané et préparé. Les difficultés les plus fréquentes pour nos publics ont été identifiées et classées.

D'un point de vue pédagogique, beaucoup de phonéticiens soutiennent qu'il faut d'abord installer l'intonation et le rythme du français avant de se concentrer sur la prononciation correcte des sons (par exemple Madeleni et al. 137). Michel Billières, un des principaux promoteurs de la méthode verbo-tonale, justifie ce choix par un constat évident : " au plan pédagogique l'intonation est la première chose que l'élève perçoit, avant même d'identifier des mots isolés ou les groupes de mots » (41-42). L'analyse des erreurs de nos étudiants confirme la primauté de la prosodie. En ce qui concerne le rythme du français (accent final, groupe rythmique, liaisons, enchaînements, chute du/ə/), nous constatons des difficultés dans tous les groupes linguistiques et dans tous les niveaux, y compris chez des étudiants pourtant B2 dans les autres compétences du français. Quant aux difficultés à produire les courbes intonatives de base du français, elles sont très répandues jusqu'au niveau B1.

Concernant les objectifs segmentaux, nous avons pris l'option de développer des séquences uniquement sur les phonèmes qui posent le plus de difficultés à nos apprenants. Pour les voyelles, il s'agit de /œ/, /ø/, $/ e /, / \varepsilon /, / y /$ et des trois nasales. Les difficultés varient selon les langues d'origine. Ainsi, pour les nasales, on 
peut observer une confusion de timbre, une non-nasalisation et/ou l'ajout d'un « $n$ ». Le /y/ peut être réalisé /u/ chez les hispanophones, /jy/ à l'initiale chez les Japonais, ou /ju/ chez certains anglophones. En ce qui concerne les consonnes, le / $\mathrm{R} /$ remporte la palme, tous niveaux et toutes langues confondus. Le / $\mathrm{z} /$ est aussi source de difficultés, essentiellement chez les hispanophones, de même que le /g/ et le /3/ (surtout intervocalique). Là encore, la diversité de notre public nécessite d'envisager différents écarts par rapport à la réalisation des phonèmes par des francophones natifs, ce qui rend le défi d'autant plus intéressant.

\subsection{Progression selon les niveaux du CECR}

Ce tableau synthétise les descripteurs du CECR relatifs à la prononciation pour chaque niveau (Conseil de l'Europe, 2001):

\begin{tabular}{|l|l|}
\hline A1 & $\begin{array}{l}\text { La prononciation d'un répertoire très limité d'expressions et de mots mémorisés est } \\
\text { compréhensible avec quelque effort pour un locuteur natif habitué aux locuteurs du } \\
\text { groupe linguistique de l'apprenant/utilisateur. }\end{array}$ \\
\hline A2 & $\begin{array}{l}\text { La prononciation est en général suffisamment claire pour être comprise malgré un } \\
\text { net accent étranger mais l'interlocuteur devra parfois faire répéter. }\end{array}$ \\
\hline B1 & $\begin{array}{l}\text { La prononciation est clairement intelligible même si un accent étranger est quelquefois } \\
\text { perceptible et si des erreurs de prononciation proviennent occasionnellement. }\end{array}$ \\
\hline B2 & La prononciation et l'intonation sont claires et naturelles. \\
\hline C1 = C2 & $\begin{array}{l}\text { Peut varier l'intonation et placer l'accent phrastique correctement afin d'exprimer de } \\
\text { fines nuances de sens. }\end{array}$ \\
\hline
\end{tabular}

Dans le volume de la collection «Recherches et applications » consacré à L'oral par tous les sens, Bento et Riquois font l'inventaire de toutes les recommandations du CECR en lien avec la phonétique (Abou Haidar et Llorca 137-147). La phonétique y est mentionnée 37 fois et apparait surtout comme un outil indispensable au service de la réalisation d'une tâche de communication. Toutefois, dans les grilles d'évaluation relatives à l'oral, la phonétique n'est jamais mentionnée. Les enseignants et les utilisateurs restent libres « de définir si l'exactitude phonétique constitue un objectif d'apprentissage immédiat ou à plus long terme » (Conseil de l'Europe 92).

En croisant les indications données dans :

- le CECR (2001)

- les référentiels construits pour les différents niveaux du CECR (Beacco et al. Niveau A1; Niveau A2 ; Niveau B1; Niveau B2)

- l'ouvrage La phonétique. Audition, prononciation, correction de Dominique Abry et Julie Veldeman-Abry - deux manuels de phonétique récents (Abry-Chalaron 2009 et 2011),

Geneviève Briet a dressé une liste des objectifs de prononciation pour les différents niveaux du CECR. Nous avons ensuite retravaillé ensemble cette liste en fonction de notre expérience, pour aboutir à un document de travail dans lequel nous avons inventorié et affiné les objectifs d'apprentissage depuis le niveau A1 jusqu'au B2 en ce qui concerne la syllabation, le groupe rythmique, l'accentuation, la continuité, l'intonation, les consonnes et les voyelles. Cet inventaire distingue perception et production, et prend en compte les paramètres de la prononciation du français à l'intérieur de la chaîne parlée (longueur du groupe rythmique, sons en contexte, débit, registre de langue).

\subsection{Contraintes et limites}

Le dispositif est conçu pour être utilisé dans le cadre de nos cours, du niveau A1 au B2. Pour les étudiants, l'apprentissage sur la plateforme restera une activité parmi d'autres. La plateforme ne constituera jamais le support principal d'un cours.

Le parcours d'apprentissage de la prononciation est développé sur Moodle. Les types d'activités possibles sont donc limités. Nous pouvons heureusement compter sur l'aide des conseillers techno-pédagogiques de l'ILV jamais à court d'idées pour triturer le code et détourner certaines activités de leur but premier. Malgré 
leur inventivité, nous sommes contraintes d'imaginer des parcours assez linéaires, et peu adaptatifs. On a vu plus haut que le diagnostic initial de la prononciation pouvait difficilement être automatisé, mais cela n'est pas gênant en soi puisque le dispositif est envisagé comme un prolongement du travail réalisé en classe avec l'aide du professeur.

Le temps et les moyens disponibles pour développer ce dispositif sont réduits. Un financement a permis d'engager une personne à mi-temps en 2016-2017, et une étudiante en Master FLE a rejoint l'équipe pour un stage de 3 mois. Le dispositif n'est pas terminé à l'échéance du financement, mais nous continuons à y travailler avec l'espoir de le tester dès 2018 auprès de nos étudiants.

\section{Objectifs et principes didactiques}

\subsection{Objectif général}

Le dispositif vise à exercer et évaluer la perception, la discrimination et la production des caractéristiques rythmiques, intonatives et phonémiques du FLE. L'étudiant ne travaillera jamais en autonomie totale. Un parcours à la carte sera établi par son professeur de français, sur la base d'un diagnostic réalisé dans le cadre du cours.

Voyons à présent quels sont les principes didactiques qui guident la poursuite de cet objectif.

\subsection{Approche « sans complexe»}

A l'origine du projet, il y a l'ouvrage La prononciation en classe que Geneviève Briet, Valérie Collige et moimême avons publié aux PUG en 2014. Nous y proposons une approche "sans complexe " de la prononciation du français, qui privilégie les métaphores, les schémas simples et les gestes pour faire comprendre les principales caractéristiques de la prononciation du français. Les activités collectives abordent le travail de la prononciation en classe d'une façon « décomplexée ", dans un esprit gai et léger, propice aux émotions positives afin de favoriser la motivation et l'apprentissage (Berdal-Masuy et Pairon ; Piccardo et Puozzo-Capron).

Nous voulons insuffler le même esprit dans le dispositif sur Moodle, ce qui ne va pas de soi vu les contraintes techniques exposées précédemment. Nous tentons de le faire au travers de documents déclencheurs attractifs et en proposant des exercices d'apprentissage qui impliquent d'autres formes d'intelligence que le verbo-linguistique : kinesthésique, intrapersonnelle, musicale.

\subsection{Prononciation en contexte}

Dans notre dispositif, l'apprentissage phonétique est contextualisé, lié aux objectifs lexicaux et morphosyntaxiques, ainsi qu'à l'environnement social des apprenants (Liakin et Liakina).

Nous avons pris le parti d'assigner un objectif culturel à tout le dispositif : la découverte des villes de la francophonie. En exerçant sa prononciation du français, l'étudiant parcourt des villes célèbres (par ex. Versailles) ou moins connues (par ex. Mons en Belgique). Chaque séquence d'activités repose sur des documents en lien avec la ville : photo, reportage, extrait de film, interview, chanson, publicité.

De plus, quand cela est possible, nous faisons des liens lexicaux et grammaticaux avec la maîtrise des caractéristiques rythmique, intonative ou phonémique du français. Par exemple, l'alternance nasalisationdénasalisation est liée à l'alternance masculin-féminin dans certains noms de nationalité (« CanadienCanadienne »); la prononciation des numéros de téléphone est couplée au découpage en groupes rythmiques, à la maîtrise de l'accent final de durée et à la réalisation d'une intonation ascendante/descendante ; l'opposition « tu/vous » est liée à la maîtrise du/y/-/u/, etc. 


\subsection{Une approche progressive}

Dans les manuels de prononciation, même récents, les exercices sont rarement reliés par un fil rouge et leur progression est souvent confuse. En analysant la place de la phonétique dans quelques manuels récents, Bento et Riquois constatent que « les activités liées à la phonétique (...) varient du segmental au suprasegmental en passant par l'orthoépique sans qu'aucune logique puisse être définie dans leur organisation » (147). A l'intérieur de chaque activité, les items se succèdent souvent de façon aléatoire. Leur seul lien est d'avoir en commun un phonème ou une opposition de phonèmes. Les virelangues sont l'exemple-type de l'exercice décontextualisé et d'un niveau de difficulté inadapté aux compétences de l'apprenant.

Au contraire, notre dispositif divise chaque objectif en sous-objectifs reliés aux niveaux du CECR (cf. supra, point 2.3). Chaque étudiant débute son entrainement par des activités adaptées à son niveau réel pour cette compétence de prononciation et peut progresser vers le niveau supérieur. Ainsi, un étudiant inscrit dans un cours B1 pourrait devoir d'abord réaliser un parcours de niveau A2 autour du/R/ si les bases phonémiques ne sont pas acquises. L'inverse est également envisageable.

Le temps consacré à l'apprentissage en ligne est de plus en plus long en fonction du niveau : nous estimons que les étudiants devront travailler sur la plateforme entre 15 minutes (objectif $A 1$ ) et 60 minutes d'affilée (objectif B2), et qu'ils exerceront au maximum 3 objectifs par semestre.

Nous essayons de retarder l'exposition à l'écrit afin de limiter aussi longtemps que possible l'influence de la graphie sur la prononciation. Sur Moodle, ce principe relève de la mission presque impossible vu que l'interface de navigation est écrite. Pour contourner l'obstacle avec les débutants (A1), toutes les consignes et les feedbacks sont donnés en anglais et la plupart des activités reposent sur des documents sonores. Si transcription il y a, elle est donnée en général en alphabet phonétique international (API), un code que les étudiants manipulent régulièrement lors des cours en présentiel. Dès le niveau $A 2$, nous faisons intervenir l'écrit. Au niveau B2, la plateforme propose des exercices qui travaillent de façon systématique les relations entre graphies et sons.

Enfin, nous établissons une progression dans l'adhésion affective au français. A la suite de Bertrand Lauret, nous pensons que pour bien prononcer une langue, il faut d'abord en accepter le rythme, la mélodie et les sons :

Plus on accepte de s'ouvrir à la différence phonétique (sans chercher à comparer avec sa / ses propre(s) langue(s) ), plus on se donne une chance de parvenir à une performance parfaite. Tout rejet face à la nouveauté (jugements esthétiques négatifs, croyances) est une preuve de refus d'ouverture, de refus d'une nouveauté qui est le quotidien de tous les locuteurs natifs. II est important d'entendre, de partager et de discuter ces jugements en classe pour permettre de les faire évoluer. (Lauret 21)

Pour cette raison, certaines séquences activent l'intelligence intrapersonnelle et débutent par des activités où les émotions, positives ou négatives, suscitées par un son sont mises au jour.

\subsection{Influences méthodologiques}

Nous ne nous revendiquons d'aucune autre école que celle du terrain. Au fil du temps, nous avons acquis une expérience des techniques de phonétique corrective qui fonctionnent en classe avec la plupart des étudiants. Les activités que nous proposons s'inspirent tant de la méthode articulatoire que de la verbotonale. Nous sommes également très influencées par l'approche gestuelle développée par Régine Llorca.

Nous puisons aussi notre inspiration en dehors du champ de la didactique de la prononciation : activités théâtrales et ludiques (Renard), pleine conscience, et théorie des intelligences multiples (Gardner).

Enfin, nous tentons d'inscrire notre façon de travailler la prononciation dans les approches actionnelles. Ainsi, certains parcours se terminent par une tâche finale qui suscite l'interaction à distance entre 
Rassart, Emmanuelle. « Concevoir un parcours d'auto-apprentissage guidé de la prononciation du FLE sur Moodle. » Nouvelle Revue Synergies Canada, №11 (2018)

participants et la co-construction du savoir (par exemple créer un nouvel item d'exercice pour les participants suivants).

\section{Architecture du dispositif et métaphores}

Ce schéma montre comment sera structuré le dispositif, à terme.

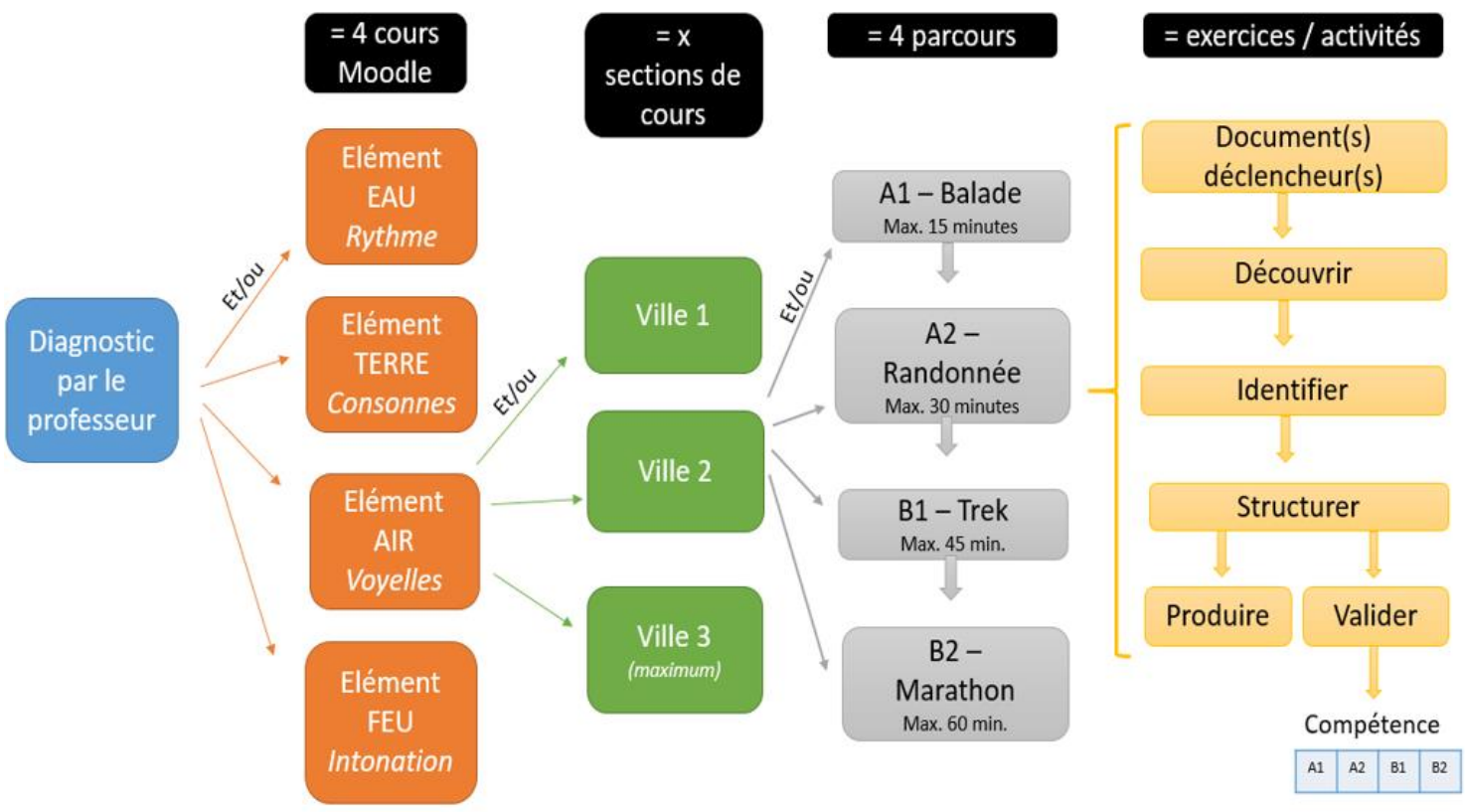

Afin de rendre plus claire l'architecture, nous avons choisi trois métaphores : les quatre éléments, les villes francophones et des promenades de difficulté variée pour découvrir ces villes.

A un premier niveau, nous lions les quatre dimensions de la prononciation (intonation, rythme, voyelles, consonnes) aux quatre éléments. Cette association n'est pas aléatoire ; elle illustre de façon «non complexe » les caractéristiques intonatives, rythmiques et phonémiques du français.

- $\quad$ Eau $=$ rythme $:$ Comme les vagues viennent se déposer inlassablement les unes après les autres sur le sable, l'accent final rythme régulièrement le flux de la parole en français. L'eau coule de façon continue et fluide, de même que les mots s'enchaînent en français à l'intérieur des groupes phonétiques. Enfin, l'eau est le liant qui donne à la pâte son onctuosité, sa souplesse, comme les mécanismes prosodiques de continuité (liaisons, enchaînements, consonnes euphoniques) assurent la cohésion du groupe phonétique.

- $\quad$ Feu = intonation : L'intonation varie en hauteur. Elle monte tant que la phrase n'est pas finie et descend à la fin, comme les flammes qui s'élèvent jusqu'à ce que le feu retombe et s'éteigne. Nous faisons aussi le lien entre le fait que l'intonation donne à la phrase son mouvement musical, son expressivité, ses nuances, et le feu qui symbolise la passion, l'énergie.

- $\quad$ Air = voyelles : Les voyelles sont à la base de la langue orale. Sans elles il n'y aurait que du bruit, pas de sons, pas de syllabes, pas d'accentuation, et donc pas de rythme. Elles sont aussi essentielles à la langue que l'air à la vie humaine.

- $\quad$ Terre = consonnes : Les consonnes sont des sons produits par l'obstruction du passage de l'air dans les cavités qui forment le canal vocal. Tous ces obstacles physiques rencontrés par l'air peuvent être imaginés comme des montagnes à gravir, des canyons étroits à franchir, des grottes profondes dans lesquelles se glisser. 
Rassart, Emmanuelle. « Concevoir un parcours d'auto-apprentissage guidé de la prononciation du FLE sur Moodle. » Nouvelle Revue Synergies Canada, №11 (2018)

A l'intérieur de chaque élément, trois ou quatre villes francophones sont proposées à la visite. Par exemple, l'élément eau associé au rythme est décliné en trois villes :

\begin{tabular}{|l|l|}
\hline Ville & Objectif \\
\hline Versailles (et ses fontaines) & Accentuation \\
\hline Ottawa (et son canal Rideau) & Continuité \\
\hline $\begin{array}{l}\text { Une ville à définir en Guadeloupe (et la mer } \\
\text { des Caraïbes) }\end{array}$ & « e » instable \\
\hline
\end{tabular}

De nouveau, chaque ville suscite des métaphores qui aident les étudiants à percevoir les caractéristiques du français. Ainsi, la continuité est liée à Ottawa. Deux métaphores aquatiques inspirent le choix de cette ville francophone et des documents et activités qui y sont proposés :

- Le Canal Rideau rythmé par ses écluses évoque le découpage en groupes rythmiques dans la chaîne parlée ;

- Le mouvement du patineur qui glisse en longues foulées sur le Canal gelé mime la façon dont la voix glisse d'une syllabe à l'autre dans le groupe rythmique.

Une fois arrivé à destination, l'apprenant découvre à pied la ville francophone en suivant une promenade adaptée à ses capacités. Quatre parcours pédestres, du plus facile au plus physique, sont proposés dans chaque ville, en lien avec les niveaux du CECR :

- $\quad$ La balade $(A 1)$

- $\quad$ La randonnée $(\mathrm{A} 2)$

- $\quad$ Le trek (B1)

- $\quad$ Le marathon (B2).

Chaque parcours pédestre est structuré en quatre ou cinq étapes : découverte du (ou des) document(s) déclencheur(s), identification de l'objet d'apprentissage, structuration de l'apprentissage, et production d'un énoncé oral plus ou moins long (répétition, imitation ou création).

Au terme de chaque promenade, l'étudiant peut passer un test certificatif pour valider son apprentissage. A chaque type de promenade dans chaque ville correspond l'octroi d'une compétence. Ceci permet de valoriser les acquis intermédiaires, même si l'objectif de «prononciation naturelle » (B2) n'est pas encore atteint.

\subsection{Exemple : parcours sur la continuité à Ottawa}

Quelques activités issues du parcours sur la continuité à Ottawa illustreront les choix didactiques décrits précédemment: la première partie de la balade sur la syllabation (niveau $A 1$ ), et la dernière partie du marathon $B 2$ intitulée « $A$ la recherche de la syllabe idéale »3.

\subsection{Balade A1 sur la syllabation (extrait)}

Outre la découverte de la capitale du Canada, l'objectif de cette première balade est de syllaber et de prendre conscience de la liaison obligatoire déterminant-nom et pronom sujet-verbe.

Comme il s'agit du niveau $\mathrm{A} 1$, les consignes et feedbacks sont donnés en anglais. En ce qui concerne les transcriptions en API, nous avons recours à un code simplifié, accessible aux non-spécialistes ${ }^{4}$.

Les documents déclencheurs de l'étape «découvrir » sont authentiques: il s'agit de photos récentes d'Ottawa. La première activité consiste à associer à ces photos des énoncés simples, comportant un seul groupe phonétique de maximum 3 syllabes. 
Rassart, Emmanuelle. « Concevoir un parcours d'auto-apprentissage guidé de la prononciation du FLE sur Moodle. » Nouvelle Revue Synergies Canada, №11 (2018)

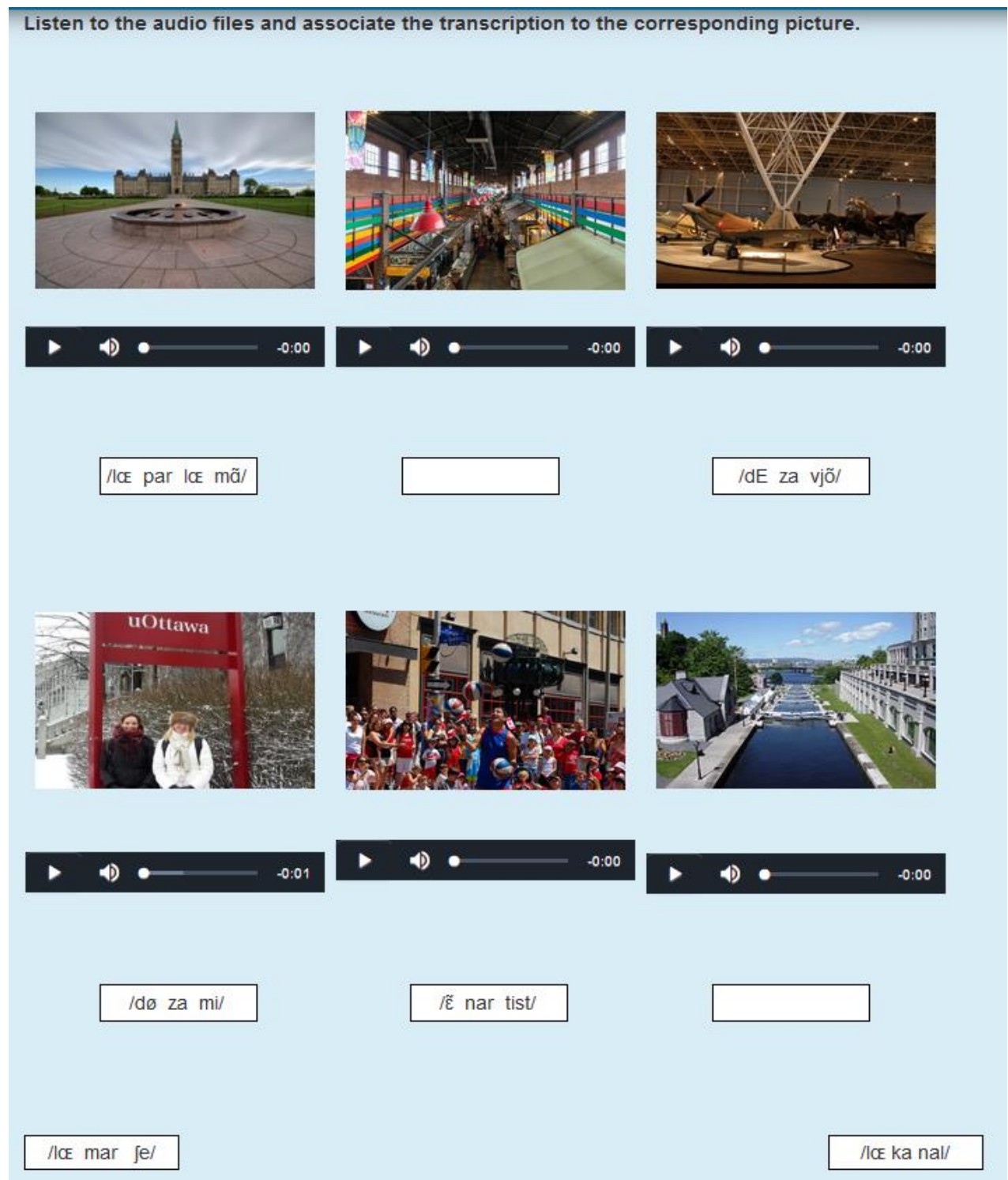

Ensuite, dans l'étape « identifier », les étudiants réécoutent certains énoncés de l'exercice précédent, et en comptent les syllabes. Pour les aider, un canevas rythmique est donné (ti ti TA). Le « TA » indique la syllabe accentuée, marquée d'un allongement caractéristique.

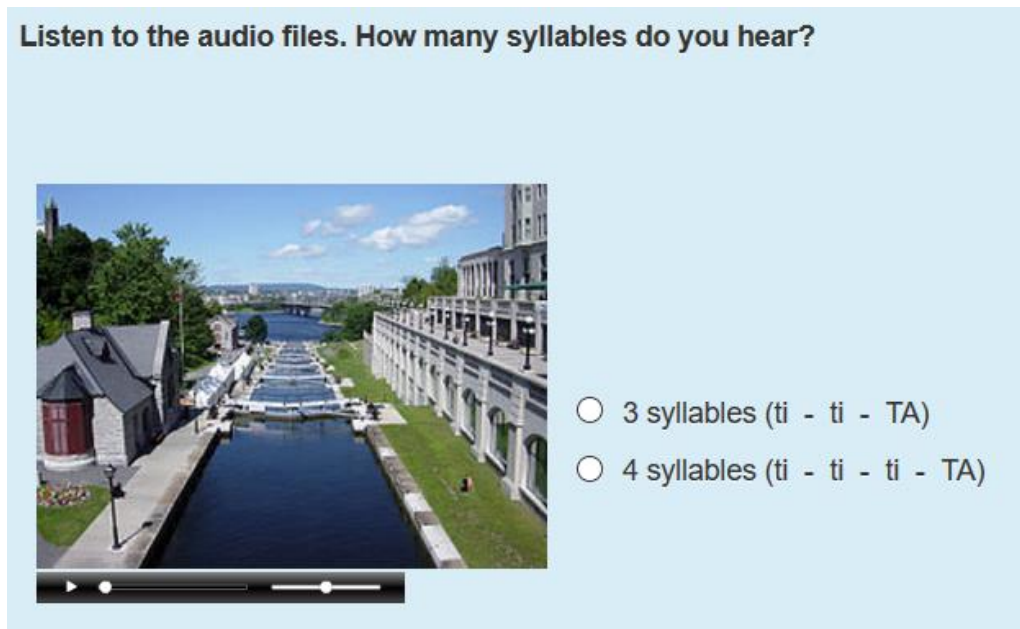


L'étape « structurer » s'ouvre sur un court tutoriel théorique qui explique comment compter les syllabes en français. Le commentaire est donné en français et des mots-clés apparaissent à l'écran. Un exercice d'application suit. Les énoncés à écouter (sans transcription orthographique) comptent cette fois au maximum 5 syllabes. Une photo d'illustration aide les étudiants à les comprendre. Le vocabulaire est simple, adapté au niveau A1. Le thème impose cependant l'introduction de quelques mots n'appartenant pas au vocabulaire de base, mais nous avons pris soin de choisir des mots transparents dans beaucoup de langues (ex. : kayak).

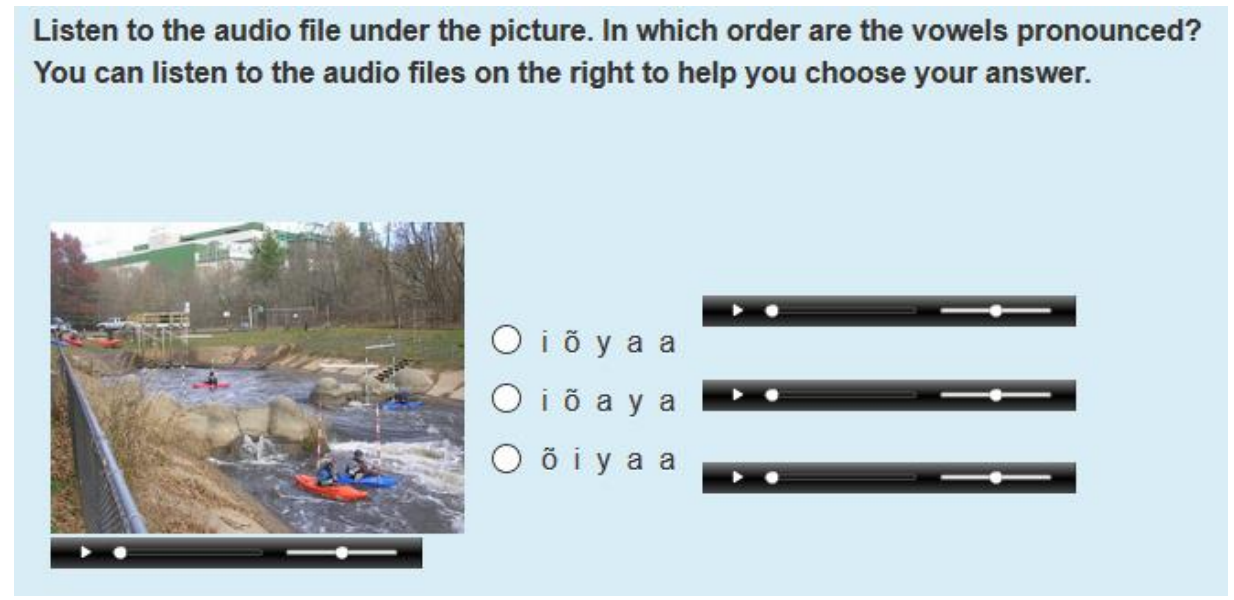

Une fois terminée cette première partie de la balade, pour laquelle 3 tentatives sont autorisées, les étudiants ont accès à une activité certificative. II n'y a pas d'étape «produire » à ce stade (début de parcours A1) vu que seule la perception est travaillée. A l'issue de l'activité certificative, les étudiants peuvent obtenir une première compétence en syllabation (niveau $\mathrm{A} 1$ ). Notons qu'ils n'ont pas été exposés directement à l'orthographe du français.

\subsection{Marathon B2 sur la continuité (extrait)}

L'objectif de cette dernière partie du marathon B2 consacré à la continuité est de découvrir deux techniques particulières du français pour obtenir dans la chaîne parlée la syllabe idéale Consonne + Voyelle (+ consonne éventuelle), notée CV(c) : l'élision de « que » et l'addition de consonnes euphoniques.

Au niveau B2, les apprenants sont censés acquérir une prononciation « naturelle » du français selon le CECR. Les documents déclencheurs relèvent donc de l'oral spontané, avec des groupes rythmiques plus longs, des spécificités prosodiques bien marquées, des accents régionaux, des voix typées, ou encore des registres de langue non standards. Nous attendons des étudiants qu'ils produisent à la fin du marathon un énoncé rythmiquement complexe.

Plusieurs documents déclencheurs sont proposés dans ce marathon qui, pour rappel, peut demander jusqu'à une heure de travail. Dans la première partie, les étudiants ont découvert quelques éléments d'histoire du Canal Rideau. Ils se sont entraînés à allonger les groupes rythmiques tout en continuant à bien connecter les syllabes et les mots lexicaux qui les composent. Pour aller plus loin dans la visite de la capitale canadienne, la dernière partie du marathon propose de faire connaissance avec le sport le plus populaire au Canada : le hockey sur glace.

L'étape « découvrir » se base sur un document audio au débit rapide et au vocabulaire très spécifique : un commentaire sportif des meilleurs moments d'un match entre les Sénateurs d'Ottawa et les Canadiens de Montréal. Les questions posées visent à faire deviner de quel sport il s'agit, et à saisir les caractéristiques rythmiques et intonatives très particulières du commentaire sportif (Boulakia et Mathon). Ces questions préparent les étudiants à la tâche de production finale qui leur sera demandée au bout du marathon. Les mots de vocabulaire utiles pour les exercices suivants sont travaillés. 
Rassart, Emmanuelle. « Concevoir un parcours d'auto-apprentissage guidé de la prononciation du FLE sur Moodle. » Nouvelle Revue Synergies Canada, №11 (2018)

L'étape « identifier » suit le fil rouge du hockey. II faut associer des extraits écrits de commentaire sportif et des photos de matchs des Sénateurs d'Ottawa. Chaque phrase a été conçue pour contenir une technique prosodique visant à créer la syllabe idéale en français $\mathrm{CV}(\mathrm{c})$. Lorsque l'étudiant a réussi l'exercice d'appariement, il reçoit un feedback dans lequel sont expliqués les procédés euphoniques présents dans chaque phrase.

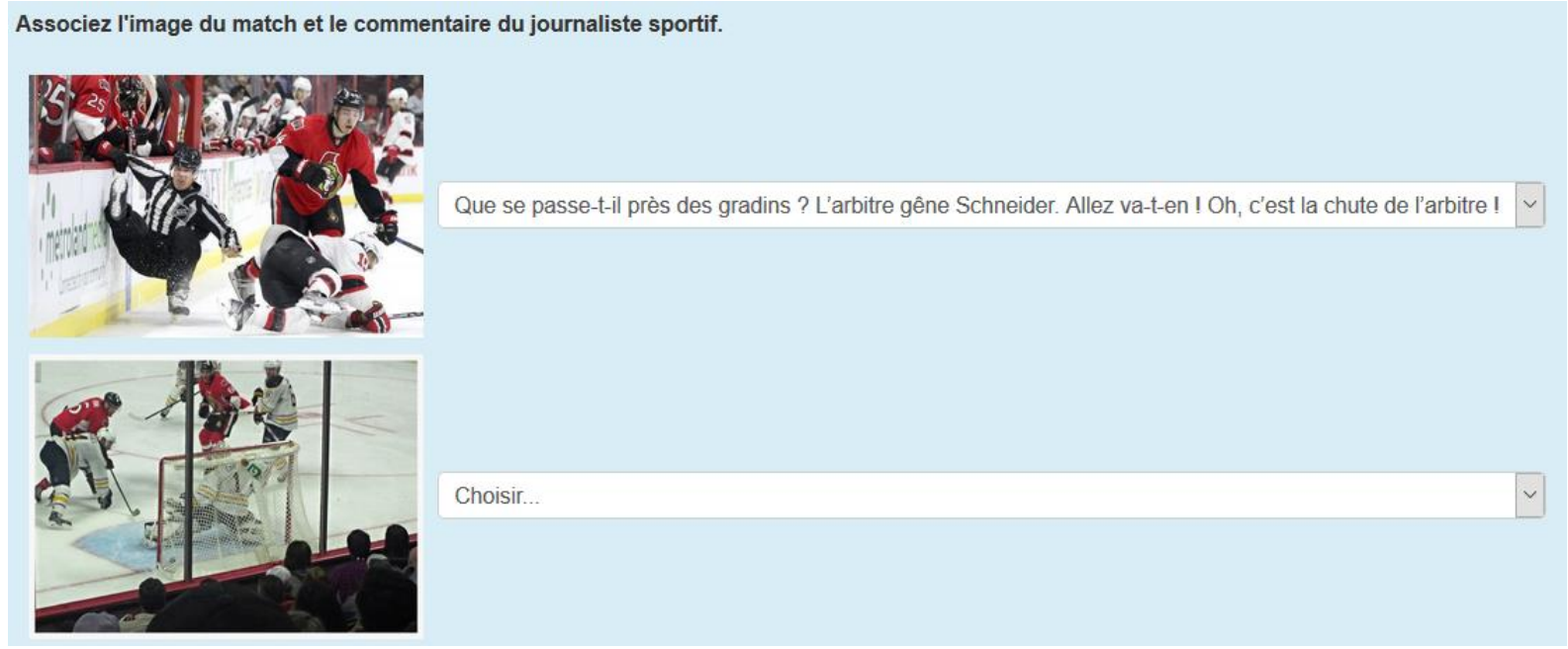

Un deuxième exercice permet d'identifier un autre procédé visant à obtenir des syllabes CV(c) : l'élision de «que ». L'étudiant est confronté à un nouveau document en lien avec le hockey : l'interview d'un coach après un match. Cette fois, l'étudiant ne peut se fier qu'à son oreille. II n'a pas accès à la transcription. Comme dans l'exercice précédent, le feedback explique les procédés présents dans les énoncés qui viennent d'être écoutés.

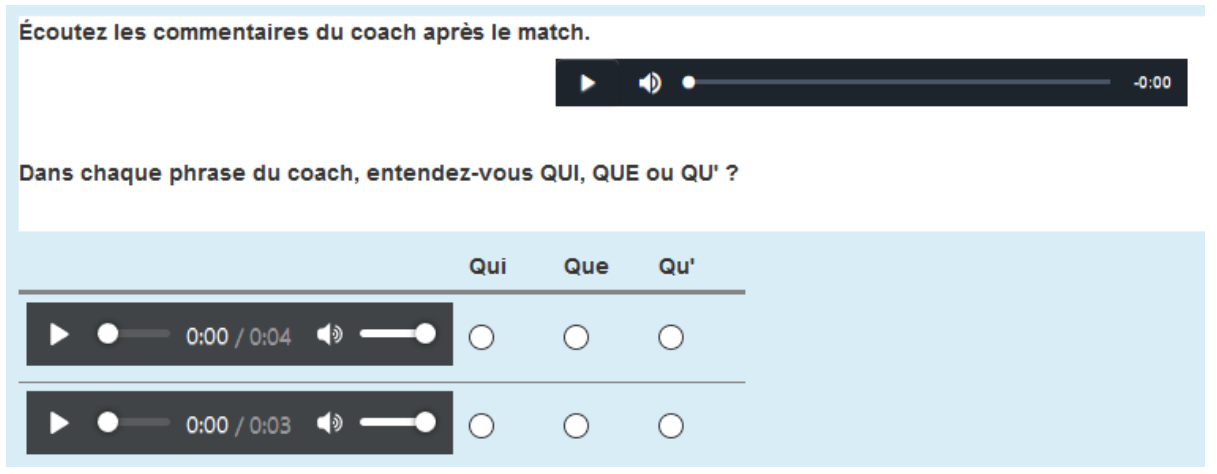

L'étape « structurer » se concentre sur les consonnes euphoniques dans les questions avec inversion verbesujet, présentes dans un registre de langue plus formel. II s'agit d'un exercice classique de reconstitution d'une question dont tous les mots et signes et ponctuation sont présentés dans le désordre. Pour rappel, selon les principes didactiques présentés plus haut, c'est au niveau B2 que sont traités les aspects complexes de la correspondance entre graphie et son. En l'occurrence, il s'agit ici de placer les traits d'union au bon endroit. Le thème des questions à reconstruire porte toujours sur le hockey sur glace. 


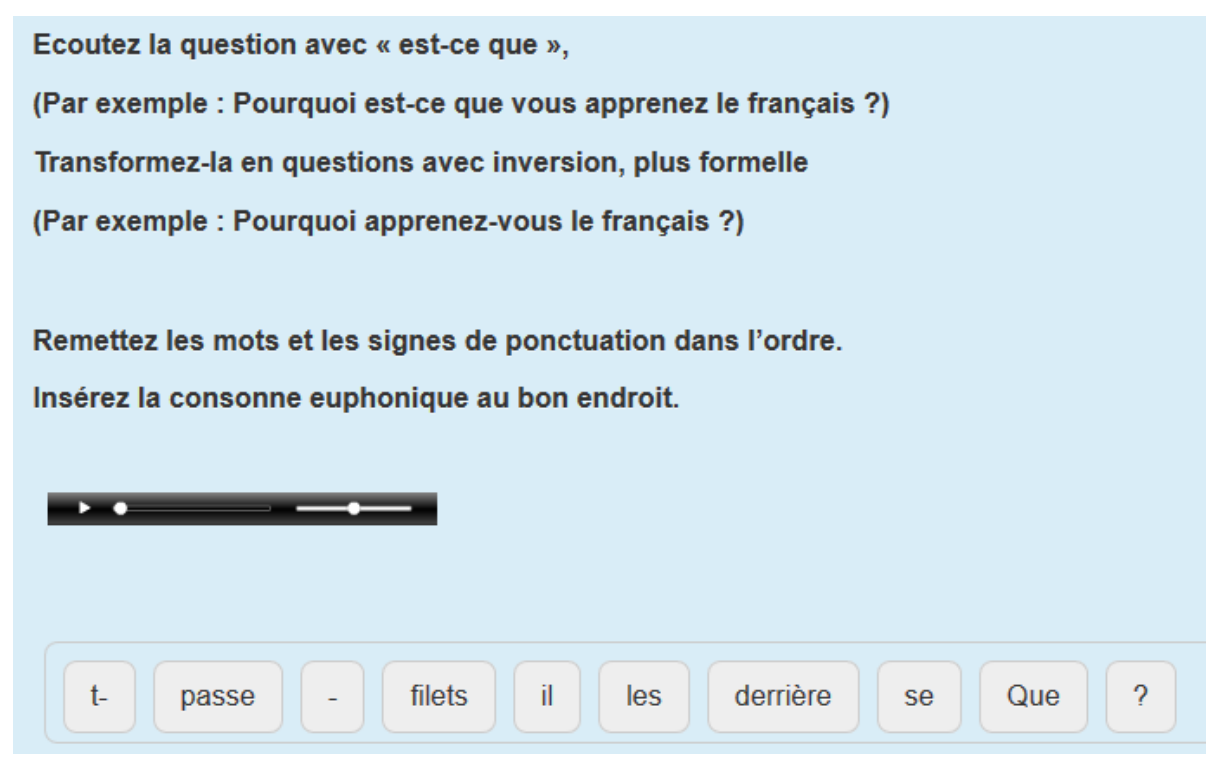

Enfin, l'étudiant va « produire » à son tour des énoncés qui contiennent les procédés euphoniques identifiés. II doit dire à voix haute les questions qui viennent d'être reconstituées en veillant à glisser de façon fluide de syllabe en syllabe. II est invité à s'enregistrer et à comparer sa production avec le modèle donné à titre indicatif.

Dans l'étape ultime de validation de la compétence « continuité B2 », il faut produire le commentaire sportif des images d'un but marqué par les Sénateurs d'Ottawa. Pour se l'approprier, les étudiants sont d'abord invités à remettre les parties du commentaire dans l'ordre en regardant les images du but. Chaque phrase contient un des procédés euphoniques qui ont été exercés auparavant. Certains groupes phonétiques sont longs (par ex. huit syllabes pour " et passe la rondelle à Turris »). Les images vont très vite, le débit doit donc être rapide. Les mouvements des joueurs filant sur la glace sont censés guider la voix des étudiants, qui glisse d'une syllabe à l'autre avec la même rapidité et la même fluidité que les hockeyeurs. C'est ce type d'activité qui permet de prolonger en ligne l'approche kinesthésique de la prononciation développée en classe. La voix du commentateur reproduit en miroir les gestes du hockeyeur.

Pour terminer, l'étudiant est invité à déposer l'enregistrement de son commentaire sur Moodle, où il pourra être écouté par les autres étudiants. Ceci donne une coloration interactionnelle au dispositif. Une fois l'enregistrement déposé, l'étudiant reçoit la compétence « continuité B2 » davantage pour son endurance que pour la qualité de sa production, vu l'impossibilité du feedback automatisé. Libre à chaque professeur d'écouter et éventuellement d'évaluer cette production finale.

\section{Conclusion}

Améliorer en ligne sa prononciation, envisagée comme rythme, intonation et phonèmes, est possible. Malgré les limites de Moodle, les étudiants peuvent s'entrainer après la classe avec des activités variées, créatives, interactives. L'approche « sans complexe » développée en groupe pendant les cours en présentiel se prolonge à distance, grâce notamment au recours aux intelligences multiples (surtout musicale, intrapersonnelle et kinesthésique), à des documents-déclencheurs riches de contenu, ou encore à des feedbacks et des tutoriels épurés le plus possible de jargon théorique.

Reste à tester ce dispositif encore en développement auprès des étudiants dès 2018. Et bien sûr, à espérer que les chercheurs en traitement automatique des langues mettent au point un outil de reconnaissance vocale capable de donner une rétroaction immédiate et valide de la prononciation du français. 
Rassart, Emmanuelle. « Concevoir un parcours d'auto-apprentissage guidé de la prononciation du FLE sur Moodle. » Nouvelle Revue Synergies Canada, №11 (2018)

\section{Notes}

1 Par exemple la découverte du « e » final non prononcé : http://apprendre.tv5monde.com/fr/apprendrefrancais/prononciation-le-e-final-non-prononce-0

2 Par exemple le jeu «Astronautes FLS » que Denis Liakin (Concordia University) a présenté lors du Colloque international sur la didactique des langues secondes, CISLS6, Université McGill, en avril 2017.

3 Les captures d'écran témoignent d'étapes intermédiaires du travail. La mise en page doit encore être améliorée.

4 Le schwa est transcrit par l'archiphonème /œE/ (cf. Lauret 65 pour une justification de l'abandon de la transcription /ə/ « didactiquement préjudiciable ») ; la durée vocalique n'est pas précisée ; la nasale « on » est transcrite /õ/ et non / $/$ /, car le /o/ permet à l'apprenant de mieux visualiser l'arrondissement de la bouche (ex. Walter 53).

\section{Bibliographie}

Abou Haidar, Laura et Régine Llorca (dir.), L'oral par tous les sens : de la phonétique corrective à la didactique de la parole. Numéro spécial de Recherches et applications. Le français dans le monde, $\mathrm{n}^{\circ} 60$, juillet 2016.

Abry, Dominique et Julie Veldeman-Abry. La phonétique. Audition, prononciation, correction. CLE International, 2007.

Abry, Dominique et Marie-Laure Chalaron. Les 500 exercices de phonétique avec corrigés A1/A2. Hachette, 2009.

---. Les 500 exercices de phonétique avec corrigés B1/B2. Hachette, 2011.

Beacco, Jean-Claude et al. Niveau B2 pour le français (utilisateur/apprenant indépendant). Un référentiel. Didier, 2004.

Beacco, Jean-Claude et Rémy Porquier. Niveau A1 pour le français (utilisateur/apprenant élémentaire). Un référentiel. Didier, 2007.

Beacco, Jean-Claude et al. Niveau A2 pour le français (utilisateur/apprenant élémentaire). Niveau intermédiaire. Un référentiel. Didier, 2008.

---. Niveau B1 pour le français (utilisateur/apprenant indépendant). Niveau seuil. Un référentiel. Didier, 2011.

Berdal-Masuy, Françoise et Christine Renard. « Comment évaluer l'impact des pratiques théâtrales sur les progrès en langue cible Renard? Vers un nouveau dispositif d'évaluation de l'oral en FLE. » Lidil, vol. 52, décembre 2015, pp. 153-174.

Berdal-Masuy, Françoise et Jacqueline Pairon. Affects et acquisition des langues. Numéro spécial de Le Langage et l'Homme, n502, 2015.

Billières, Michel. « Le corps en phonétique corrective. » Apprentissage d’une langue

étrangère/seconde, dir. Renard Raymond, De Boeck Université, 2002, pp. 37-70.

---. "Méthode articulatoire et méthode verbo-tonale." Phonétique corrective en FLE, 2013, http://w3.uohprod.univ-tlse2.fr/UOH-PHONETIQUE-FLE/seq04P0101.html. Accès 5 avril 2017.

Boulakia, Georges et Catherine Mathon. « Le commentaire sportif en direct : une combinatoire de différentes fonctions de la prosodie. " H-Y Yoo, et E. Delais-Roussarie (éd.). Actes d'IDP 2009, Paris, 9-11 septembre 2009, 2011, pp. 287-301. http://makino.linguist.jussieu.fr/idp09/actes fr.html. Accès le 10 juillet 2017.

Briet, Geneviève et al. La prononciation en classe. Presses universitaires de Grenoble, 2014. 
Rassart, Emmanuelle. « Concevoir un parcours d'auto-apprentissage guidé de la prononciation du

FLE sur Moodle. » Nouvelle Revue Synergies Canada, №11 (2018)

Briet, Geneviève. «Développer l'ego phonétique par une didactique de l'émotionnel ». Le Langage et I'Homme, n49/1, 2014, pp. 25-35.

Cardoso, Walcir et al. "Learning L2 pronunciation with a mobile speech recognizer: French /y/. » Calico, vol. 32.1, 2014, pp. 1-25.

Chapelle, Carol A. et Joan Jamieson. Tips for Teaching with CALL: Practical Approaches to ComputerAssisted Language Learning. Pearson-Longman, 2008.

Charliac, Lucile et Annie-Claude Motron. Phonétique progressive du français. Niveau avancé. CLE International, 2006.

Collige, Valérie. «La prononciation en classe : apprendre ensemble avec le corps, en 'pleine présence.' » Affects et acquisition des langues, Berdal-Masuy, Françoise et Pairon, Jacqueline (dir.). Numéro spécial de Le langage et l'homme, 50/2, 2015.

Conseil de l'Europe. Cadre européen commun de référence pour les langues. Didier, 2001.

Dufeu, Bernard. «Vers une approche holistique de la prononciation. » L'oral par tous les sens : de la phonétique corrective à la didactique de la parol, Laura Abou Haidar et Régine Llorca (dir.). Recherches et applications. Le français dans le monde, 60, 2016, pp. 58-69.

Gardner, Howard. Les Intelligences Multiples. Retz, 2005.

Lauret, Bertrand. Enseigner la prononciation du français : questions et outils. Hachette, 2007.

Liakin, Denis et Natallia Liakina. «Applications pour l'apprentissage de la prononciation du français : quel impact? Quel potentiel ? " Communication orale présentée lors du XIVe congrès mondial des professeurs de français à Liège, 14-21 juillet 2016.

Llorca, Régine. «Du geste à la parole. » Francparler.org, 2008, http://www.francparleroif.org/images/stories/articles/llorca2008.htm\#q1. Accès le 5 avril 2017.

Madeleni, Edith et al. Le rythme du français parlé. Hachette, 2012.

Piccardo, Enrica et Isabelle Puozzo Capron. « L'émotion et l'apprentissage des langues. » Lidil, 48, 2013.

Renard, Christine. "Les activités ludiques en classe de FLE : l'art d'instruire et d'apprendre avec plaisir. 》 Français 2000, 212/213, avril 2008, pp. 66-54.

Walter, Henriette. La phonologie du français. Phonologies des usages du français, 1977. 